Annales Geophysicae, 23, 2589-2597, 2005

SRef-ID: 1432-0576/ag/2005-23-2589

(C) European Geosciences Union 2005

\title{
Relation between magnetic fields and electric currents in plasmas
}

\author{
V. M. Vasyliūnas \\ Max-Planck-Institut für Sonnensystemforschung, 37191 Katlenburg-Lindau, Germany
}

Received: 16 May 2005 - Revised: 26 June 2005 - Accepted: 27 June 2005 - Published: 14 October 2005

\begin{abstract}
Maxwell's equations allow the magnetic field $\boldsymbol{B}$ to be calculated if the electric current density $\boldsymbol{J}$ is assumed to be completely known as a function of space and time. The charged particles that constitute the current, however, are subject to Newton's laws as well, and $\boldsymbol{J}$ can be changed by forces acting on charged particles. Particularly in plasmas, where the concentration of charged particles is high, the effect of the electromagnetic field calculated from a given $\boldsymbol{J}$ on $\boldsymbol{J}$ itself cannot be ignored. Whereas in ordinary laboratory physics one is accustomed to take $\boldsymbol{J}$ as primary and $\boldsymbol{B}$ as derived from $\boldsymbol{J}$, it is often asserted that in plasmas $\boldsymbol{B}$ should be viewed as primary and $\boldsymbol{J}$ as derived from $\boldsymbol{B}$ simply as $(c / 4 \pi) \nabla \times \boldsymbol{B}$. Here I investigate the relation between $\nabla \times \boldsymbol{B}$ and $\boldsymbol{J}$ in the same terms and by the same method as previously applied to the MHD relation between the electric field and the plasma bulk flow (Vasyliūnas, 2001): assume that one but not the other is present initially, and calculate what happens. The result is that, for configurations with spatial scales much larger than the electron inertial length $\lambda_{e}$, a given $\nabla \times \boldsymbol{B}$ produces the corresponding $\boldsymbol{J}$, while a given $\boldsymbol{J}$ does not produce any $\nabla \times \boldsymbol{B}$ but disappears instead. The reason for this can be understood by noting that $\nabla \times \boldsymbol{B} \neq(4 \pi / c) \boldsymbol{J}$ implies a time-varying electric field (displacement current) which acts to change both terms (in order to bring them toward equality); the changes in the two terms, however, proceed on different time scales, light travel time for $\boldsymbol{B}$ and electron plasma period for $\boldsymbol{J}$, and clearly the term changing much more slowly is the one that survives. (By definition, the two time scales are equal at $\lambda_{e}$.) On larger scales, the evolution of $\boldsymbol{B}$ (and hence also of $\nabla \times \boldsymbol{B}$ ) is governed by $\nabla \times \boldsymbol{E}$, with $\boldsymbol{E}$ determined by plasma dynamics via the generalized Ohm's law; as illustrative simple examples, I discuss the formation of magnetic drift currents in the magnetosphere and of Pedersen and Hall currents in the ionosphere.
\end{abstract}

Keywords. Ionosphere (Electric fields and currents) Magnetospheric physics (Magnetosphere-ionosphere interactions) - Space plasma physics (Kinetic and MHD theory)

Correspondence to: V. M. Vasyliūnas

(vasyliunas@mps.mpg.de)

\section{Introduction}

Starting with elementary courses in electromagnetism, one becomes accustomed to think of the magnetic field $\boldsymbol{B}$ as determined by the given distribution of electric current density $\boldsymbol{J}$. Many researchers, particularly in magnetospheric physics, continue to apply this mode of thinking (that $\boldsymbol{B}$ is derived from $\boldsymbol{J}$ ) when investigating problems of large-scale plasma physics, for which the converse view - that $\boldsymbol{J}$ is derived from $\boldsymbol{B}$ simply as $(c / 4 \pi) \nabla \times \boldsymbol{B}$ - has been proposed by Cowling (1957) and Dungey (1958) and in recent years strongly argued by Parker $(1996,2000)$. From Maxwell's equations (Gaussian units are used throughout this paper)

$$
\begin{aligned}
& \partial \boldsymbol{B} / \partial t=-c \nabla \times \boldsymbol{E}, \\
& \partial \boldsymbol{E} / \partial t=-4 \pi \boldsymbol{J}+c \nabla \times \boldsymbol{B}, \\
& \nabla \cdot \boldsymbol{B}=0 \quad \nabla \cdot \boldsymbol{E}=4 \pi \rho_{c}
\end{aligned}
$$

one easily obtains an equation explicitly relating $\boldsymbol{B}$ to $\boldsymbol{J}$ only:

$$
\left(1 / c^{2}\right) \partial^{2} \boldsymbol{B} / \partial t^{2}-\nabla^{2} \boldsymbol{B}=(4 \pi / c) \nabla \times \boldsymbol{J},
$$

allowing $\boldsymbol{B}$ at any point to be derived from a knowledge of $\boldsymbol{J}$ over the complete backward light cone of the point (plus knowledge of boundary conditions at $-\infty$ ). When dealing with a plasma, however, there are two plasma equations involving $\boldsymbol{J}$ that must be considered in addition to Maxwell's equations: the generalized Ohm's law (see, e.g. Vasyliūnas, 2005 , and references therein)

$$
\begin{gathered}
\partial \boldsymbol{J} / \partial t=\sum_{a}\left\{\left(q_{a}^{2} n_{a} / m_{a}\right)\left(\boldsymbol{E}+\boldsymbol{V}_{a} \times \boldsymbol{B} / c\right)\right. \\
\left.-\left(q_{a} / m_{a}\right) \nabla \cdot \boldsymbol{\kappa}_{a}+q_{a} n_{a} \boldsymbol{g}\right\}+(\delta \boldsymbol{J} / \delta t)_{\text {coll }}
\end{gathered}
$$

representing in its exact form (Eq. 5) the charge-weighted summation of all particle accelerations, and the plasma momentum equation

$\partial \rho \boldsymbol{V} / \partial t+\nabla \cdot \boldsymbol{\kappa}=\boldsymbol{J} \times \boldsymbol{B} / c+\rho \boldsymbol{g}+\boldsymbol{f}$.

In Eq. (5), $q_{a}, m_{a}, n_{a}, \boldsymbol{V}_{a}$, and $\boldsymbol{\kappa}_{a}$ are the charge, mass, concentration, bulk velocity, and kinetic tensor, respectively, of particle species $a,(\delta \boldsymbol{J} / \delta t)_{\text {coll }}$ represents the sum of all collision effects, and $\boldsymbol{g}$ is the gravitational acceleration (included 
for exactness but mostly unimportant in practice). Equation (6) contains the parameters of the plasma as a whole: $\rho$ is the mass density, $\boldsymbol{V}$ is the bulk velocity, and

$\kappa=\sum_{a} \kappa_{a}=\rho \boldsymbol{V} \boldsymbol{V}+\mathrm{P}$

is the kinetic tensor; $\boldsymbol{f}$ stands for any other force, not explicitly displayed.

When the full set of Eqs. (1-6) is to be taken into account, it is by no means obvious that a complete specification of $\boldsymbol{J}$, prior to and independently of $\boldsymbol{B}$, is possible. If we are dealing with a situation where the displacement current may be neglected and Eq. (2) reduces to the ordinary Ampère's law,

$\boldsymbol{J}=(c / 4 \pi) \nabla \times \boldsymbol{B}$,

then the two sides of the equation must be equal, but the question remains: which side is the one that can be specified first (and hence taken as producing the other)?

This paper attempts to determine whether and under what conditions a definite physical answer to the above question can be given. It is the third paper in a series dealing with some fundamental questions, motivated in part by the controversy (Parker, 1996, 1997, 2000; Heikkila, 1997; Lui, 2000) on whether $\boldsymbol{B}$ and $\boldsymbol{V}$ or, instead, $\boldsymbol{J}$ and $\boldsymbol{E}$ are to be treated as the primary variables. The first paper (Vasyliūnas, 2001) considered an analogous question: given that $\boldsymbol{V}$ and $\boldsymbol{E}$ are connected by the MHD approximation, which one can be regarded as producing the other? The clear answer was that, provided the Alfvén speed $V_{A}^{2} \ll c^{2}$ (i.e. the inertia of the plasma is dominated by the rest mass of the plasma particles and not by the relativistic energy-equivalent mass of the magnetic field), $\boldsymbol{V}$ produces $\boldsymbol{E}$ but not vice versa. The second paper (Vasyliūnas, 2005) showed that, on space and time scales larger than those of electron plasma oscillations, the time evolution of $\boldsymbol{J}$ cannot be calculated directly but only as the time evolution of $(c / 4 \pi) \nabla \times \boldsymbol{B}$. In this paper I show that this subordination of $\boldsymbol{J}$ to $(c / 4 \pi) \nabla \times \boldsymbol{B}$ holds not just for the time derivatives but also for the quantities themselves.

\section{Relation between fields and currents evolved from ini- tial values}

Questions of the type raised here and in Vasyliūnas (2001) are sometimes phrased in terms of causes: which term is the cause of which? Put this way, however, the discussion may wander off too easily into issues of philosophy, a subject in which physicists as such have no particular competence (how many physicists have a clear idea, for instance, of the distinction between efficient and formal cause?) and which is unlikely to lead to an unambiguous statement of physical results. For this reason I have avoided as much as possible the notion of cause and have tried to reduce everything to specific questions about observable physical effects: which term (when assumed to exist initially) implies (through the equations) the development of which?

\subsection{The initial-value thought experiment method}

What makes this approach possible is the evolutionary character of the basic equations of classical (non-quantum) physics (Vasyliūnas, 2001, 2005), which allows an arbitrary configuration, subject only to the constraint of satisfying the divergence equations (3) (plus the analogous gravitational equation), to be postulated at an initial time $t=0$, the equations then specifying completely the evolution of the system at all other times. Thus, to investigate the question, which of the two quantities $\boldsymbol{J}$ and $(c / 4 \pi) \nabla \times \boldsymbol{B}$ determines the other, we may assume at $t=0$ any initial spatial profile of $\boldsymbol{J}$ and $\boldsymbol{B}$, subject to the sole constraint that $\nabla \cdot \boldsymbol{B}=0$ but not satisfying Eq. (7), and then follow their evolution for $t>0$ by solving Eqs. (1-6).

\subsection{Vacuum case}

It is instructive to consider first the case of a system in a vacuum, where only Maxwell's equations need to be solved. Assume that at $t=0$ the initial magnetic field has $\nabla \times \boldsymbol{B} \neq 0$, even though $\boldsymbol{J}=0$ everywhere. Obviously, the non-curl-free magnetic field will propagate away at the speed of light, accompanied by appropriate electric fields as required by Maxwell's equations.

As a simple example, consider a 1-D magnetic field reversal:

$$
\boldsymbol{B}=\operatorname{sign}(z) B_{0} \hat{\boldsymbol{x}} \quad \boldsymbol{E}=0
$$

at $t=0$ in a vacuum, with no current to support the reversal. At all later times $t>0$, the magnetic and electric fields are given by

$$
\begin{array}{llll}
\boldsymbol{B}=0 & \boldsymbol{E}=B_{0} \hat{\boldsymbol{y}} & & |z|<c t \\
\boldsymbol{B}=\operatorname{sign}(z) B_{0} \hat{\boldsymbol{x}} & \boldsymbol{E}=0 & & |z|>c t .
\end{array}
$$

The initial field reversal plane splits into two planes, propagating away at the speed of light in the $\pm \hat{z}$ directions, at which the initial $\boldsymbol{B}$ is replaced by $\boldsymbol{E}$ in the perpendicular direction; it is easily checked that this satisfies Maxwell's equations. (The apparent persistence of $\boldsymbol{E}$ at $|z|<c t$ for all times is an artifact of neglecting the finite extent of the system.)

Note that if a plasma were present, $\boldsymbol{E}$ given by Eq. (9) would drive a current in the sense required to support the initial reversal of $\boldsymbol{B}$. Simply inserting this $\boldsymbol{E}$ into the generalized Ohm's law (5) would give

$\boldsymbol{J} \approx\left(n_{e} e^{2} / m_{e}\right) \boldsymbol{E} t$

With the current density increasing as $\sim t$ and current layer thickness $z=c t$, the current per unit length soon reaches the value that would have maintained the initial field reversal in a steady state:

$$
(4 \pi / c) \boldsymbol{J} z \approx B_{0} \hat{\boldsymbol{y}} \quad \text { when } \quad z=c t=c / \omega_{p} \equiv \lambda_{e},
$$

where $\omega_{p}^{2}=4 \pi \sum_{a} q_{a}^{2} n_{a} / m_{a} \approx 4 \pi n_{e} e^{2} / m_{e}$ is the (electron) plasma frequency and $\lambda_{e}$ the electron inertial length. Of 
course, combining a vacuum solution of Maxwell's equations with the generalized Ohm's law is not consistent, and this calculation does not prove anything. It does, however, suggest that, when Eq. (7) is not satisfied, changes occur on temporal and spatial scales of order of $1 / \omega_{p}$ and $\lambda_{e}$, and the important terms in the generalized Ohm's law (5) are $\partial \boldsymbol{J} / \partial t$ and $\left(n_{e} e^{2} / m_{e}\right) \boldsymbol{E}$; this is consistent with the results of a more rigorous analysis (Vasyliūnas, 2005).

\subsection{Plasma case}

Now take initial values $\boldsymbol{B}_{0}(\boldsymbol{r}), \boldsymbol{E}_{0}(\boldsymbol{r})$, and $\boldsymbol{J}_{0}(\boldsymbol{r})$, arbitrary except for satisfying whatever spatial boundary conditions apply and for the constraint $\nabla \cdot \boldsymbol{B}=0$, and solve Maxwell's equations, together with the generalized Ohm's law, which can be written (following Vasyliūnas, 2005) as

$\partial \boldsymbol{J} / \partial t=\left(\omega_{p}^{2} / 4 \pi\right)\left(\boldsymbol{E}-\boldsymbol{E}^{*}\right)$,

$\boldsymbol{E}^{*}$ standing for all the terms except the first on the righthand side of Eq. (5); the discussion above suggests that it be approximated by the reduced form

$\partial \boldsymbol{J} / \partial t \approx\left(\omega_{p}^{2} / 4 \pi\right) \boldsymbol{E}$

presumed valid for the temporal and spatial scales involved, of order of $1 / \omega_{p}$ and $\lambda_{e}$ (the implications of this assumption and the consequences of including the $\boldsymbol{E}^{*}$ term are discussed in Sect. 2.5).

Initial value problems are conveniently solved by introducing the Laplace transform in time:

$\tilde{Q}(\boldsymbol{r}, s)=\int_{0}^{\infty} d t \mathrm{e}^{-s t} Q(\boldsymbol{r}, t)$,

the transform of the time derivative bringing in the initial value $Q_{0} \equiv Q(\boldsymbol{r}, 0)$

$\int_{0}^{\infty} d t \mathrm{e}^{-s t} \partial Q / \partial t=s \tilde{Q}-Q_{0}$.

The transformed Eqs. (1), (2), and (13) are

$s \tilde{\boldsymbol{B}}-\boldsymbol{B}_{0}=-c \nabla \times \tilde{\boldsymbol{E}}$,

$s \tilde{\boldsymbol{E}}-\boldsymbol{E}_{0}=-4 \pi \tilde{\boldsymbol{J}}+c \nabla \times \tilde{\boldsymbol{B}}$,

$s \tilde{\boldsymbol{J}}-\boldsymbol{J}_{0}=\left(\omega_{p}^{2} / 4 \pi\right) \tilde{\boldsymbol{E}}$.

An equation for $\tilde{\boldsymbol{B}}$ alone is obtained by taking the curl of Eq. (17) and using Eqs. (16) and (18) to eliminate $\tilde{\boldsymbol{E}}$ and $\tilde{\boldsymbol{J}}$ :

$$
\begin{gathered}
-\lambda_{e}{ }^{2} \nabla^{2} \tilde{\boldsymbol{B}}+\left(1+s^{2} / \omega_{p}{ }^{2}\right) \tilde{\boldsymbol{B}}=\left(1 / s+s / \omega_{p}{ }^{2}\right) \boldsymbol{B}_{0} \\
+\lambda_{e}{ }^{2} \nabla \times\left[(4 \pi / c) \boldsymbol{J}_{0} / s-\boldsymbol{E}_{0} / c\right] .
\end{gathered}
$$

Similarly, an equation for $\tilde{\boldsymbol{J}}$ alone is obtained by taking the curl of the curl of Eq. (18) and using Eqs. (16), (17), and (18) to eliminate $\tilde{\boldsymbol{E}}$ and $\widetilde{\boldsymbol{B}}$ :

$$
\begin{aligned}
\lambda_{e}{ }^{2} \nabla & \times \nabla \times \tilde{\boldsymbol{J}}+\left(1+s^{2} / \omega_{p}{ }^{2}\right) \tilde{\boldsymbol{J}}=\left(s / \omega_{p}{ }^{2}\right) \boldsymbol{J}_{0} \\
& +\lambda_{e}{ }^{2} \nabla \times \nabla \times \boldsymbol{J}_{0} / s+(c / 4 \pi)\left[\nabla \times \boldsymbol{B}_{0} / s+\boldsymbol{E}_{0} / c\right] .
\end{aligned}
$$

Finally, an equation for $\tilde{\boldsymbol{E}}$ alone is obtained by using Eq. (18) and the curl of Eq. (16) to eliminate $\tilde{\boldsymbol{J}}$ and $\tilde{\boldsymbol{B}}$ in Eq. (17):

$$
\begin{aligned}
\lambda_{e}{ }^{2} \nabla \times \nabla \times \tilde{\boldsymbol{E}}+\left(1+s^{2} / \omega_{p}{ }^{2}\right) \tilde{\boldsymbol{E}} & = \\
\left(1 / \omega_{p}{ }^{2}\right) & {\left[s \boldsymbol{E}_{0}-4 \pi \boldsymbol{J}_{0}+c \nabla \times \boldsymbol{B}_{0}\right] . }
\end{aligned}
$$

As a check, note that if the initial values $\boldsymbol{B}_{0}, \boldsymbol{J}_{0}$ satisfy Eq. (7) and $\boldsymbol{E}_{0}=0$, the solutions of Eqs. (19), (20), and (21) are easily shown to be

$$
\tilde{\boldsymbol{B}}=\boldsymbol{B}_{0} / s \quad \tilde{\boldsymbol{J}}=\boldsymbol{J}_{0} / s \quad \tilde{\boldsymbol{E}}=0
$$

which are the Laplace transforms of

$$
\boldsymbol{B}=\boldsymbol{B}_{0} \quad \boldsymbol{J}=\boldsymbol{J}_{0} \quad \boldsymbol{E}=0,
$$

i.e. as expected, the initial values do not change.

Equations (19), (20), and (21), solved subject to the applicable spatial boundary conditions, give the Laplace transforms $\tilde{\boldsymbol{B}}(\boldsymbol{r}, s), \tilde{\boldsymbol{J}}(\boldsymbol{r}, s), \tilde{\boldsymbol{E}}(\boldsymbol{r}, s)$; these can be inverted to obtain $\boldsymbol{B}(\boldsymbol{r}, t), \boldsymbol{J}(\boldsymbol{r}, t), \boldsymbol{E}(\boldsymbol{r}, t)$ by the standard technique of contour integration in the complex $s$ plane. Of primary interest, however, are the mean values for times $t \gg 1 / \omega_{p}-$ formally, the limit $t \rightarrow \infty$. In the contour integration, they are given by the residues of the pole at $s=0$; thus, for any quantity $Q$

$Q_{\infty}(\boldsymbol{r})=\lim _{s \rightarrow 0} s \tilde{Q}(\boldsymbol{r}, s)$.

Other singularities, at $|s| \sim O\left(\omega_{p}\right)$, will contribute either decaying or oscillatory terms.

Taking the limit defined by expression (24) in Eqs. (19), (20), and (21) gives

$$
\begin{aligned}
& \boldsymbol{B}_{\infty}-\lambda_{e}{ }^{2} \nabla^{2} \boldsymbol{B}_{\infty}=\boldsymbol{B}_{0}+\lambda_{e}{ }^{2} \nabla \times(4 \pi / c) \boldsymbol{J}_{0}, \\
& \boldsymbol{J}_{\infty}+\lambda_{e}{ }^{2} \nabla \times \nabla \times \boldsymbol{J}_{\infty}= \\
& (c / 4 \pi) \nabla \times \boldsymbol{B}_{0}+\lambda_{e}{ }^{2} \nabla \times \nabla \times \boldsymbol{J}_{0},
\end{aligned}
$$

and

$$
\boldsymbol{E}_{\infty}+\lambda_{e}{ }^{2} \nabla \times \nabla \times \boldsymbol{E}_{\infty}=0
$$

as the equations to be solved to obtain the final values $\boldsymbol{B}_{\infty}$, $\boldsymbol{J}_{\infty}, \boldsymbol{E}_{\infty}$ into which the assumed initial values $\boldsymbol{B}_{0}, \boldsymbol{J}_{0}, \boldsymbol{E}_{0}$ evolve. Equations (25) and (26) also imply that

$$
\left\{1+\lambda_{e}{ }^{2} \nabla \times \nabla \times\right\}\left[\boldsymbol{J}_{\infty}-(c / 4 \pi) \nabla \times \boldsymbol{B}_{\infty}\right]=0 .
$$

Equations (27) and (28) are independent of initial values; hence, to order $\left(\lambda_{e} / \mathcal{L}\right)^{2}, \boldsymbol{E}_{\infty}$ vanishes and $\boldsymbol{B}_{\infty}$ and $\boldsymbol{J}_{\infty}$ satisfy Ampère's law, for any initial values. For the rest, it is evident that if the initial values vary spatially only on scales $\mathcal{L} \gg \lambda_{e}$, Eqs. (25) and (26) reduce to

$$
\begin{aligned}
& \boldsymbol{B}_{\infty}=\boldsymbol{B}_{0}+O\left(\lambda_{e} / \mathcal{L}\right)^{2} \\
& \boldsymbol{J}_{\infty}=(c / 4 \pi) \nabla \times \boldsymbol{B}_{0}+O\left(\lambda_{e} / \mathcal{L}\right)^{2} .
\end{aligned}
$$

$\boldsymbol{B}$ maintains its initial value, while $\boldsymbol{J}$ becomes equal to $(c / 4 \pi) \nabla \times \boldsymbol{B}$ regardless of its own initial value. 
Explicit solutions of Eqs. (25), (26), and (27) can be written down for the 1-D geometry

$$
\boldsymbol{B}=B(z, t) \hat{\boldsymbol{x}} \quad \boldsymbol{J}=J(z, t) \hat{\boldsymbol{y}} \quad \boldsymbol{E}=E(z, t) \hat{\boldsymbol{y}}
$$

with initial values

$B(z, 0)=B_{0}(z) \quad J(z, 0)=J_{0}(z) \quad E(z, 0)=0$

$\left(d B_{0} / d z \neq(4 \pi / c) J_{0}\right)$ and spatial boundary condition of all quantities vanishing at $z \rightarrow \pm \infty$. The solutions for $t \rightarrow \infty$ are: $E=0$ and

$$
\begin{aligned}
B & =\left\langle B_{0}\right\rangle-(4 \pi / c) \lambda_{e}{ }^{2}\left\langle d J_{0} / d z\right\rangle, \\
J & =(c / 4 \pi)\left\langle d B_{0} / d z\right\rangle-\lambda_{e}{ }^{2}\left\langle d^{2} J_{0} / d z^{2}\right\rangle \\
& =(c / 4 \pi)\left\langle d B_{0} / d z\right\rangle+\left(J_{0}-\left\langle J_{0}\right\rangle\right),
\end{aligned}
$$

where

$$
\langle\xi(z)\rangle \equiv \frac{1}{2} \int_{-\infty}^{\infty} \frac{d z^{\prime}}{\lambda_{e}} \xi\left(z^{\prime}\right) \exp \left\{-\left(\frac{\left|z-z^{\prime}\right|}{\lambda_{e}}\right)\right\}
$$

is a spatial average around a point, heavily weighted to the neighborhood within a distance $\lambda_{e}$ from it; the second line of Eq. (34) follows from the first by two integrations by parts.

\subsubsection{Alternative direct derivation}

Equations (25), (26), and (27) can also be derived without explicit use of the Laplace transform. For $\boldsymbol{B}_{\infty}$, take the time derivative of Eq. (4), replace the resulting $\partial \boldsymbol{J} / \partial t$ on the righthand side from Eq. (13) and the resulting $\nabla \times \boldsymbol{E}$ from Eq. (1) to obtain

$\partial / \partial t\left[\left(1 / c^{2}\right) \partial^{2} \boldsymbol{B} / \partial t^{2}-\nabla^{2} \boldsymbol{B}\right]=-\left(\omega_{p}{ }^{2} / c^{2}\right) \partial \boldsymbol{B} / \partial t$.

Integrating Eq. (36) with respect to $t$ from 0 to $\infty$ gives

$$
\begin{aligned}
\left(1 / c^{2}\right) & {\left[\left(\partial^{2} \boldsymbol{B} / \partial t^{2}\right)_{\infty}-\left(\partial^{2} \boldsymbol{B} / \partial t^{2}\right)_{0}\right] } \\
- & \nabla^{2}\left(\boldsymbol{B}_{\infty}-\boldsymbol{B}_{0}\right)=-\left(\omega_{p}^{2} / c^{2}\right)\left(\boldsymbol{B}_{\infty}-\boldsymbol{B}_{0}\right),
\end{aligned}
$$

$\left(\partial^{2} \boldsymbol{B} / \partial t^{2}\right)_{0}$ can be evaluated in terms of initial values from Eq. (4), and $\left(\partial^{2} \boldsymbol{B} / \partial t^{2}\right)_{\infty}$ is assumed zero. Inserting these values into Eq. (37) and rearranging terms gives Eq. (25).

For $\boldsymbol{J}_{\infty}$, the electric-field counterpart of Eq. (4) is needed:

$$
\left(1 / c^{2}\right) \partial^{2} \boldsymbol{E} / \partial t^{2}+\nabla \times \nabla \times \boldsymbol{E}=-\left(4 \pi / c^{2}\right) \partial \boldsymbol{J} / \partial t .
$$

Apply the differential operator on the left-hand side of Eq. (38) to Eq. (13) and use Eq. (38) to replace the righthand side, to obtain

$$
\begin{gathered}
{\left[\left(1 / c^{2}\right) \partial^{2} / \partial t^{2}+\nabla \times \nabla \times\right] \partial \boldsymbol{J} / \partial t=} \\
-\left(\omega_{p}{ }^{2} / c^{2}\right) \partial \boldsymbol{J} / \partial t .
\end{gathered}
$$

Integrating Eq. (39) with respect to $t$ from 0 to $\infty$ gives

$$
\begin{aligned}
& \left(1 / c^{2}\right)\left[\left(\partial^{2} \boldsymbol{J} / \partial t^{2}\right)_{\infty}-\left(\partial^{2} \boldsymbol{J} / \partial t^{2}\right)_{0}\right] \\
& +\nabla \times \nabla \times\left(\boldsymbol{J}_{\infty}-\boldsymbol{J}_{0}\right)=-\left(\omega_{p}^{2} / c^{2}\right)\left(\boldsymbol{J}_{\infty}-\boldsymbol{J}_{0}\right) .
\end{aligned}
$$

Differentiating Eq. (13) with respect to time and applying Eq. (2) gives

$$
\partial^{2} \boldsymbol{J} / \partial t^{2}=\left(\omega_{p}^{2} / 4 \pi\right)(c \nabla \times \boldsymbol{B}-4 \pi \boldsymbol{J}),
$$

from which $\left(\partial^{2} \boldsymbol{J} / \partial t^{2}\right)_{0}$ can be evaluated in terms of initial values; $\left(\partial^{2} \boldsymbol{J} / \partial t^{2}\right)_{\infty}$ is assumed zero, as before. With this, Eq. (40) can be rearranged to give Eq. (26).

For $\boldsymbol{E}_{\infty}$, taking Eq. (38) at $t \rightarrow \infty$, inserting Eq. (13) for $\partial \boldsymbol{J} / \partial t$, and neglecting $\left(\partial^{2} \boldsymbol{E} / \partial t^{2}\right)_{\infty}$ gives Eq. (27) directly.

Although this alternative derivation may appear simpler, one crucial step, neglecting the second time derivatives at $t \rightarrow \infty$, can be properly justified only by the Laplacetransform treatment.

\subsection{Physical description}

Underlying the complicated-looking mathematics of Sect. 2.3 is a simple physical picture. Fundamentally, it is the motion of all the charged particles in a volume that constitutes the electric current in that volume. If the curl of the magnetic field does not equal the current density determined by the charged-particle motion (factors of $4 \pi / c$ taken as understood throughout this discussion), Maxwell's equations say that an electric field will develop, having a curl that implies a change in the magnetic field in turn, tending to make the curl of the magnetic field equal to the current density; the whole process takes place at the speed of light. These changing electric and magnetic fields, however, also change the motion of charged particles and hence affect the current density, tending to make it equal to the curl of the magnetic field; the time scale of this process is the period of electron plasma oscillations.

Which way an initially imposed difference between $\boldsymbol{J}$ and $(c / 4 \pi) \nabla \times \boldsymbol{B}$ resolves itself depends thus on the ratio of two times scales: light travel time across a typical spatial scale $\mathcal{L}$ vs. the inverse of the electron plasma frequency $\omega_{p}$. When $\mathcal{L} / c \ll 1 / \omega_{p}$ (or $\left.\mathcal{L} \ll c / \omega_{p} \equiv \lambda_{e}\right), \boldsymbol{J}$ hardly changes during the time interval it takes for $\boldsymbol{B}$ to reach adjustment with Ampère's law, Eq. (7). It is then convenient to treat $\boldsymbol{J}$ as given and to calculate $\boldsymbol{B}$ (neglecting the usually ignorable retardation effects) from Eq. (7); this is the environment of the elementary EM laboratory with its circuits and devices. In space and astrophysical plasmas, on the other hand, typically $\mathcal{L} \gg \lambda_{e}$; then $\boldsymbol{J}$ can change to satisfy Ampère's law in a time interval during which $\boldsymbol{B}$ hardly changes. Equation (7) can now be used only to calculate $\boldsymbol{J}$ from $(c / 4 \pi) \nabla \times \boldsymbol{B}$; the magnetic field itself must be determined from other considerations (some examples are discussed in Sect. 3).

\subsection{Plasma response and magnetic field evolution}

So far the evolution of $\boldsymbol{B}$ and $\boldsymbol{J}$ from given initial values has been calculated assuming that direct acceleration of charged particles by the electric field is the dominant mechanism for changing the current on the short $\left(\sim 1 / \omega_{p}\right)$ time scales involved - the assumption underlying the reduction of the generalized Ohm's law to the approximate form Eq. (13). The 
consequences of all the other field and plasma effects that change the current, contained in the $\boldsymbol{E}^{*}$ term of Eq. (12), can be studied by including that term (treated formally as a given function of space and time) when taking the Laplace transforms. The transformed Eqs. (16) and (17) remain unchanged while Eq. (18) is replaced by

$s \tilde{\boldsymbol{J}}-\boldsymbol{J}_{0}=\left(\omega_{p}^{2} / 4 \pi\right)\left(\tilde{\boldsymbol{E}}-\tilde{\boldsymbol{E}}^{*}\right)$,

from which it follows that the previously derived Eqs. (19), (20), and (21) remain valid provided one substitutes

$\boldsymbol{J}_{0} \rightarrow \boldsymbol{J}_{0}-\left(\omega_{p}{ }^{2} / 4 \pi\right) \tilde{\boldsymbol{E}}^{*}$

whenever $\boldsymbol{J}_{0}$ appears in the equations. Applying the limit defined by expression (24), needed to obtain the long-time solutions, to the substitution (43) requires some care because of the singularities of $\tilde{\boldsymbol{E}}^{*}$ at $s \rightarrow 0$. Using integration by parts and the definition of the Laplace transform as needed, one can show that

$$
\begin{gathered}
\lim _{s \rightarrow 0} s\left[\boldsymbol{J}_{0}-\left(\omega_{p}^{2} / 4 \pi\right) \tilde{\boldsymbol{E}}^{*}\right]=\left(\omega_{p}^{2} / 4 \pi\right) \boldsymbol{E}_{\infty}^{*}, \\
\lim _{s \rightarrow 0} s\left\{\left[\boldsymbol{J}_{0}-\left(\omega_{p}^{2} / 4 \pi\right) \tilde{\boldsymbol{E}}^{*}\right] / s\right\} \\
=\boldsymbol{J}_{0}-\left(\omega_{p}{ }^{2} / 4 \pi\right) \int_{0}^{\infty} d t \boldsymbol{E}^{*}(\boldsymbol{r}, t), \\
\lim _{s \rightarrow 0} s\left\{\left[\boldsymbol{J}_{0}-\left(\omega_{p}{ }^{2} / 4 \pi\right) \tilde{\boldsymbol{E}}^{*}\right] s\right\} \\
=-\left(\omega_{p}{ }^{2} / 4 \pi\right)\left(\partial \boldsymbol{E}^{*} / \partial t\right)_{\infty} .
\end{gathered}
$$

With the use of expression (44) in place of $\boldsymbol{J}_{0}$ in Eq. (21), the equation for $\boldsymbol{E}_{\infty}$, replacing Eq. (27), becomes

$\boldsymbol{E}_{\infty}+\lambda_{e}{ }^{2} \nabla \times \nabla \times \boldsymbol{E}_{\infty}=\boldsymbol{E}_{\infty}^{*}$.

The equation for $\boldsymbol{B}_{\infty}$ retains the form of Eq. (25) if $\boldsymbol{J}_{0}$ in it is replaced by the expression (45). Noting that only the curl of $\boldsymbol{J}_{0}$ appears, one may carry out what looks at first like a purely mathematical simplification: given $\boldsymbol{E}^{*}(\boldsymbol{r}, t)$, define a field $\boldsymbol{B}^{*}(\boldsymbol{r}, t)$ as the solution of the equation

$\partial \boldsymbol{B}^{*} / \partial t=-c \nabla \times \boldsymbol{E}^{*}$

subject to the boundary condition $\boldsymbol{B}^{*}(\boldsymbol{r}, 0)=\boldsymbol{B}_{0}(\boldsymbol{r})$. Then, with the use of expression (45), the curl of the substitution (43) (multiplied by $\lambda_{e}^{2}$ for convenience) can be rewritten as

$\lambda_{e}^{2} \nabla \times \boldsymbol{J}_{0} \rightarrow \lambda_{e}^{2} \nabla \times \boldsymbol{J}_{0}+(c / 4 \pi)\left(\boldsymbol{B}_{\infty}^{*}-\boldsymbol{B}_{0}\right)$

and inserted into Eq. (25) gives

$\boldsymbol{B}_{\infty}-\lambda_{e}{ }^{2} \nabla^{2} \boldsymbol{B}_{\infty}=\boldsymbol{B}_{\infty}^{*}+\lambda_{e}{ }^{2} \nabla \times(4 \pi / c) \boldsymbol{J}_{0}$.

Finally, the equation for $\boldsymbol{J}_{\infty}$ differs from Eq. (26) both in replacing $\boldsymbol{J}_{0}$ by expression (45), handled as above, and in having an added term, arising out of the first term on the right-hand side of Eq. (20), equal to expression (46) divided by $\omega_{p}{ }^{2}$. The resulting equation is

$$
\begin{aligned}
\boldsymbol{J}_{\infty} & +\lambda_{e}{ }^{2} \nabla \times \nabla \times \boldsymbol{J}_{\infty}=(c / 4 \pi) \nabla \times \boldsymbol{B}_{\infty}^{*} \\
& -(1 / 4 \pi)\left(\partial \boldsymbol{E}^{*} / \partial t\right)_{\infty}+\lambda_{e}{ }^{2} \nabla \times \nabla \times \boldsymbol{J}_{0} .
\end{aligned}
$$

The physical meaning of the procedure is now apparent Provided the spatial variations of $\boldsymbol{E}^{*}$ and of the initial values $\boldsymbol{B}_{0}, \boldsymbol{J}_{0}$, and $\boldsymbol{E}_{0}$ are only on scales $\mathcal{L} \gg \lambda_{e}$, the solutions of Eqs. (47), (50), and (51) are, to order $\left(\lambda_{e} / \mathcal{L}\right)^{2}$,

$\boldsymbol{E}_{\infty}=\boldsymbol{E}_{\infty}^{*}$

$\boldsymbol{B}_{\infty}=\boldsymbol{B}_{\infty}^{*}$,

$\boldsymbol{J}_{\infty}=(c / 4 \pi) \nabla \times \boldsymbol{B}_{\infty}^{*}-(1 / 4 \pi)\left(\partial \boldsymbol{E}^{*} / \partial t\right)_{\infty}$,

i.e. the asymptotic mean values, reached for times $\tau \gg 1 / \omega_{p}$, are determined solely by $\boldsymbol{E}_{\infty}^{*}$ and $\boldsymbol{B}_{\infty}^{*}$ and do not depend at all on the initial values. But it is a property of plasmas on large spatial and temporal scales

$\mathcal{L} \gg \lambda_{e} \quad$ and $\quad \tau \gg 1 / \omega_{p}$

that the generalized Ohm's law can be reduced to

$0 \approx\left(\omega_{p}^{2} / 4 \pi\right)\left(\boldsymbol{E}-\boldsymbol{E}^{*}\right)$

(Vasyliūnas, 2005), determining the electric field which then governs, via Maxwell's equations, the evolution of the magnetic field and hence the value of the current. Thus, $\boldsymbol{E}^{*}$ equals the electric field and $\boldsymbol{B}^{*}$ is the magnetic field, both calculated from approximate equations valid in the large-scale plasma limit (55) discussed by Vasyliūnas (2005). What Eqs. (52), (53), and (54) show is that the electromagnetic fields and currents calculated from the exact evolutionary equations with given initial values for all the quantities become, on time scales longer than the electron plasma oscillation period (and provided the initial values satisfy the large-scale condition), indistinguishable from those calculated from the approximate equations.

\section{Examples of magnetic field evolution}

For plasmas on scales large in the sense of inequalities (55), only $\nabla \times \boldsymbol{B}$ can be independently specified and not $\boldsymbol{J}$ : if (unconstrained) initial values for both are assumed, $\boldsymbol{J}$ evolves within a time of order of $1 / \omega_{p}$ to the value required by Ampère's law while $\nabla \times \boldsymbol{B}$ remains essentially unchanged. Since this notion - that $\boldsymbol{B}$ determines $\boldsymbol{J}$ rather than the other way around - runs counter to so much conventional thinking, it is useful to illustrate it by some specific examples. In the following, I consider a few cases where a priori specification of the current might seem "intuitively obvious" and, by tracing the evolution from initial conditions, show that in fact the current develops from deformation of the magnetic field by plasma dynamics.

\subsection{Magnetic drift currents}

Charged-particle drifts in inhomogeneous magnetic fields are very well known; they are, for instance, generally considered the primary contributors to the ring current in the magnetosphere during magnetic storms. If a population of moderately energetic ions were suddenly placed on a shell of dipole field lines, filling it, shouldn't a current carried by gradient and 
curvature drifts appear (on a time scale something like an ion gyroperiod), with $\boldsymbol{J}$ given by ion concentration times drift speed and $\nabla \times \boldsymbol{B}$ adjusting itself accordingly?

To obtain the current density, however, one must consider all the motions of all the charged particles. A fundamental result derived by Parker (1957) is that $\boldsymbol{J}$ obtained by summing all the single-particle drifts satisfies the plasma momentum equation, rewritten from Eq. (6) as

$\partial \rho \boldsymbol{V} / \partial t+\nabla \cdot(\rho \boldsymbol{V} \boldsymbol{V}+\mathbf{P})=\boldsymbol{J} \times \boldsymbol{B} / c$.

Gradient, curvature, and magnetization drifts add up to the pressure tensor term; the time-derivative and inertial terms come from the so-called polarization drifts (see, e.g. Northrop, 1963); and $\boldsymbol{E} \times \boldsymbol{B}$ drifts, of course, carry no net current. (The gravitational term $\rho \boldsymbol{g}$ has been neglected as unimportant in most plasma contexts; it can be derived by summing the currents carried by the $\boldsymbol{g} \times \boldsymbol{B}$ drifts.)

Consider a simple model of the symmetric ring current: ions with isotropic pressure, electrons with negligible pressure, over a region of finite radial extent. The asymptotic steady state of this configuration is well known: eastward (for the Earth's dipole field orientation) current at the inner edge (where $\partial P / \partial r>0$ ), larger westward current at the outer edge $(\partial P / \partial r<0)$, current carried by ions (in the frame of reference where $\boldsymbol{E}=0$ ), hence corresponding eastward and westward plasma bulk flows. Of interest is to trace the process by which this steady state is reached if the following initial state is assumed at $t=0: \boldsymbol{J}=0, \nabla \times \boldsymbol{B}=0$ (the magnetic field is purely dipolar), $\boldsymbol{E}=0$ (in the chosen frame of reference), and the plasma ion pressure is enhanced (within a radially limited shell of field lines), with a velocity distribution function that is purely isotropic in the $\boldsymbol{E}=0$ frame (consistent with $\boldsymbol{J}=0$ and implying also $\boldsymbol{V}=0$ ). (Slow loss processes, e.g. precipitation or charge exchange, are ignored.)

The evolution of the system is governed by Faraday's law (1), Ampère's law (7) (neglect of the displacement current, implied by the use of Eq. (7) instead of Eq. (2), is easily shown to be equivalent to neglect of $B^{2} / 4 \pi c^{2}$ in comparison to $\rho$ in the momentum equation, valid as long as $V_{A}^{2} / c^{2} \ll 1$ ), the momentum equation (57) with isotropic pressure

$\rho(\partial \boldsymbol{V} / \partial t+\boldsymbol{V} \cdot \nabla \boldsymbol{V})+\nabla P=\boldsymbol{J} \times \boldsymbol{B} / c$

(supplemented by continuity and energy equations as needed), and the generalized Ohm's law, which can be approximated by

$0=\boldsymbol{E}+\boldsymbol{V} \times \boldsymbol{B} / c-\boldsymbol{J} \times \boldsymbol{B} / n_{e} e c$

with neglect of any initial transients on times scales $O\left(1 / \omega_{p}\right)$, electron pressure, and $O\left(m_{e} / m_{i}\right)$ terms. Equations (1) and (59) can be combined immediately to give

$\partial \boldsymbol{B} / \partial t=\nabla \times\left[\boldsymbol{V} \times \boldsymbol{B}-\left(\boldsymbol{J} \times \boldsymbol{B} / n_{e} e\right)\right]$

which does not refer to $\boldsymbol{E}$ explicitly. Solving this set of equations is in general very difficult, but the various time scales can be estimated by order-of-magnitude arguments (e.g. Vasyliūnas, 1996).
At $t=0$, the only term in all these equations that is out of balance is the radial pressure gradient. Equation (58) then implies that plasma begins to flow radially, the flow velocity initially increasing linearly with time:

$V_{r} \sim(1 / \rho)(\partial P / \partial r) t$.

Given the radial $\boldsymbol{V}$, Eq. (59) implies an azimuthal $\boldsymbol{E}$, with a non-zero curl that implies a time-varying $\boldsymbol{B}$. The change in $\boldsymbol{B}$ can be estimated directly from Eq. (60):

$\delta B \sim \int d t V_{r} B / \mathcal{L} \sim(1 / \rho)(\partial P / \partial r)(B / \mathcal{L}) t^{2}$

with $\nabla \times$ represented simply as $1 / \mathcal{L}$. It is only the curl of $\delta \boldsymbol{B}$ from Eq. (62) that finally gives rise to the (azimuthal) current density, which initially increases as $t^{2}$ :

$J_{\phi} \sim(c / 4 \pi) \delta B / \mathcal{L} \sim[(c / B) \partial P / \partial r]\left(V_{A}^{2} t^{2} / \mathcal{L}^{2}\right)$.

As $\boldsymbol{J} \times \boldsymbol{B} / c$ increases, it approaches and ultimately reaches balance with $\nabla P$, putting an end to the radial flow of the plasma and thus stopping the further increase of $\delta \boldsymbol{B}$ and $J_{\phi}$. The equilibrium value of $J_{\phi}$ is equal to the quantity in [ ] on the right-hand side of Eq. (63); the order of magnitude of the time scale to reach balance is therefore $\mathcal{L} / V_{A}$, the Alfvén wave travel time across a typical spatial gradient. (The actual approach to stress balance is likely to be oscillatory.)

Injecting a population of (potentially) drifting ions thus does not by itself create a current: the initial effect is a radial displacement of the plasma by the imposed pressure imbalance, and it is only as the magnetic field becomes deformed by the plasma bulk flow that its curl gives rise to the current. The current density reaches its full drift value only after an elapsed time comparable to the Alfvén wave travel time, as the magnetic stresses approach balance with the pressure gradients.

But this is not the end of the story yet, because drifting ions imply a sustained azimuthal bulk flow of the plasma, whereas all the plasma bulk flows discussed here so far have been radial and transient. Given the azimuthal $\boldsymbol{J}$ as above and no azimuthal $\boldsymbol{V}$, Eq. (59) implies a radial $\boldsymbol{E}$, the curl of which could in principle be zero. In that case, in the chosen frame of reference (in which $\boldsymbol{E}=0$ initially) the current is carried entirely by the electrons - the gradient, curvature, and magnetization drifts of the ions are offset by $\boldsymbol{E} \times \boldsymbol{B}$ drifts from (inhomogeneous) electric fields that have appeared as part of the dynamical evolution. To change this situation requires $\nabla \times\left(\boldsymbol{J} \times \boldsymbol{B} / n_{e} e\right) \neq 0$, which from Eq. (59) allows a non-zero $B_{\phi}$ to evolve: a $B_{\phi}$ that in general varies along the field line (e.g. it may have opposite signs on the two sides of the magnetic equatorial plane) and thus gives (through the magnetic curvature force) an azimuthal component to the magnetic stress in the momentum equation, with consequent azimuthal acceleration of plasma bulk flow. (The equivalent conventional description in terms of currents is that $B_{\phi}$ varying along the field line implies a radial component of $\boldsymbol{J}$ and hence an azimuthal component of $\boldsymbol{J} \times \boldsymbol{B} / c$.) 
One obvious source of the non-zero curl is the increased electron concentration $n_{e}$ at and near the ionosphere, unrelated to the magnetospheric plasma pressure so that

$(\boldsymbol{J} \times \boldsymbol{B}) \times \nabla n_{e} \sim c \nabla P \times \nabla n_{e} \neq 0$.

Representing $\nabla \times$ as $1 / \mathcal{L}_{z}$ (to emphasize that the relevant length scale now is predominantly along the magnetic field line), we can estimate $B_{\phi}$ from Eq. (60)

$B_{\phi} \sim \int d t J_{\phi} B / n_{e} e \mathcal{L}_{z}$

and $V_{\phi}$ from Eq. (58)

$V_{\phi} \sim \int d t B B_{\phi} / 4 \pi \rho \mathcal{L}_{z}$

With the use of Eqs. (63) and (64), the estimated azimuthal bulk flow becomes

$n_{e} e V_{\phi} \sim[(c / B) \partial P / \partial r]\left(V_{A}{ }^{4} t^{4} / \mathcal{L}^{2} \mathcal{L}_{z}{ }^{2}\right)$,

written in this form for direct comparison with $J_{\phi}$ in Eq. (63), the difference $V_{\phi}-\left(J_{\phi} / n_{e} e\right)$ being what determines the radial component of $\boldsymbol{E}$ from Eq. (59). Equation (66) shows that $n_{e} e V_{\phi}$ increases from zero initially as $t^{4}$ and is at the beginning much smaller than $J_{\phi}$ which increases as $t^{2}$. Thus, relative to the chosen frame of reference, the ions indeed undergo almost no net drift at first, the current being carried mostly by the electrons. Only after an elapsed time comparable to $\mathcal{L}_{z} / V_{A}$ has the azimuthal plasma flow been accelerated to $V_{\phi} \approx\left(J_{\phi} / n_{e} e\right)$, the electric field has decreased to zero, and the ions now do carry the current as envisaged in the simple drift picture.

Another way of looking at this process is to note that isotropic pressure is constant along a field line, hence the curl of the magnetic field deformed by plasma pressure gradients varies in a smooth, well-defined manner along the field line. If the current is carried predominantly by electrons, the rapid increase in electron concentration near and in the ionosphere implies a corresponding decrease in the electron bulk velocity required to carry the current. But Eq. (59) implies, as is well known, that the magnetic field is frozen to the bulk flow of electrons. If the electrons carrying the azimuthal current are flowing much more slowly in the ionosphere than in the magnetosphere, the field lines become bent, with the curvature being in the sense to accelerate the plasma in the direction of the current (opposite to the electron flow).

The question may be raised: How is the magnetic drift current carried entirely by the ions established if there is no ionosphere? The simple answer is: It isn't! As long as the condition $\nabla P \times \nabla n_{e}=0$ can be maintained, the asymptotic steady-state solution of Eqs. (58) and (59) is given by

$\nabla P=\boldsymbol{J} \times \boldsymbol{B} / c \quad \boldsymbol{V}=0 \quad \boldsymbol{E}=\nabla P / n_{e} e ;$

the current implied by the deformation of the magnetic field continues to be carried entirely by the electrons, and the ion bulk flow remains zero - a configuration sustainable because the associated inhomogeneous electric field has zero curl. It is by interaction with the ionosphere, as described above, that eventually the azimuthal flow of the ions is established and the inhomogenous electric field eliminated. This also resolves an apparent paradox: If the initial state has, as assumed, zero bulk flow and hence zero angular momentum about the magnetic dipole axis, where does the angular momentum of the drifting ions in the final state come from? Obviously, it has been transferred from the ionosphere (and ultimately, of course, from the atmosphere and the planet) during the transient phase of non-zero $B_{\phi}$.

\subsection{Pedersen and Hall currents}

A recurrent question in studies of magnetosphere-ionosphere interaction is how the horizontal electric currents in the ionosphere evolve in response to temporal changes within the magnetosphere and the solar wind. Traditionally, the change in the electric field (or else of the plasma bulk flow) just above the ionosphere is taken as given by magnetospheric processes, and the corresponding electric current is calculated by treating the ionosphere as a resistive medium, with the resistivity due primarily to collisions between plasma and neutral particles and hence with $\boldsymbol{J}$ proportional to the electric field in the frame of reference of the neutral atmosphere. While there is hardly any doubt concerning the numerical results, there are some subtle points concerning the physical interpretation.

Consider, for simplicity, the region of the ionosphere above an altitude of $\sim 120 \mathrm{~km}$, where only ion-neutral collisions are significant and electron collisions can be neglected. The plasma momentum equation (horizontal components only) then simplifies to

$\rho \partial \boldsymbol{V} / \partial t=\boldsymbol{J} \times \boldsymbol{B} / c+v_{i n} \rho\left(\boldsymbol{V}_{n}-\boldsymbol{V}\right)$,

where $v_{i n}$ is the ion-neutral collision frequency, $\boldsymbol{V}_{n}$ is the bulk velocity of the neutral atmosphere, and the kinetic tensor terms (usually assumed unimportant in the present context) have been dropped. The generalized Ohm's law (see Vasyliūnas and Song, 2005, and references therein for a more detailed discussion) is still well approximated by Eq. (59); only at altitudes below $\sim 100 \mathrm{~km}$ do electron collision terms become important.

The first point to note is that the electric field by itself does almost nothing: in the ionosphere, $v_{i n} \ll \omega_{p}$ and therefore the result derived by Vasyliūnas (2001), that $\boldsymbol{V}$ produces $\boldsymbol{E}$ but $\boldsymbol{E}$ does not produce $\boldsymbol{V}$, applies - although his calculation did not include the effect of collisions, the adjustments he describes occur on a time scale of electron plasma frequency, so the presence or absence of ion-neutral collisions makes no difference. To study the development of currents in the ionosphere one must thus posit an initial condition of plasma flow.

Assume then that at $t=0$ there is bulk flow of plasma (both in the ionosphere and above it in the magnetosphere) relative to the frame of reference of the neutrals, but no electric current: $\boldsymbol{V} \neq \boldsymbol{V}_{n}, \boldsymbol{J}=0$. If one assumes also $\boldsymbol{E}=0$ at $t=0$, the 
electric field goes from zero to the value $-\boldsymbol{V} \times \boldsymbol{B} / c$ (consistent with Eq. (59) when $\boldsymbol{J}=0$ ) on the very short time scale of $1 / \omega_{p}$ (Vasyliūnas, 2001), so one may equally well assume this as the initial value of $\boldsymbol{E}$. In either case, the further evolution proceeds as described below.

With $\boldsymbol{J}=0$ initially, the plasma-neutral collisional friction term in Eq. (68) is not balanced, and the plasma flow is decelerated (relative to the neutrals) on a time scale $1 / v_{i n}$ :

$\delta V \sim-v_{\text {in }}\left(V-V_{n}\right) t$.

The strong altitude dependence of $1 / \nu_{\text {in }}$ leads to a corresponding altitude dependence of the deceleration (effectively, the plasma slows down in the ionosphere but not in the magnetosphere), implying from Eq. (60) a changing magnetic field:

$\delta B \sim \int d t \delta V B / \mathcal{L}_{z} \sim-v_{\text {in }}\left(V-V_{n}\right)\left(B / \mathcal{L}_{z}\right) t^{2}$,

the curl of which gives rise to the current density:

$$
\begin{aligned}
J \sim & (c / 4 \pi) \delta B / \mathcal{L}_{z} \\
& \sim\left[\left(V-V_{n}\right) c \rho v_{i n} / B\right]\left(V_{A}{ }^{2} t^{2} / \mathcal{L}_{z}{ }^{2}\right) .
\end{aligned}
$$

Initially $J$ increases as $t^{2}$ and ultimately, after a time comparable to $\mathcal{L}_{z} / V_{A}$, reaches the value in [ ] on the right-hand side of Eq. (71), at which the $\boldsymbol{J} \times \boldsymbol{B} / \boldsymbol{c}$ force and the plasmaneutral collisional friction are in balance, with no further deceleration of bulk flow. In the steady-state limit, Eq. (59) and Eq. (68) with $\partial \boldsymbol{V} / \partial t=0$ may be combined to eliminate $\boldsymbol{V}$ and express $\boldsymbol{J}$ as a linear function of $\boldsymbol{E}+\boldsymbol{V}_{n} \times \boldsymbol{B} / c$, with Pedersen and Hall conductivities - the conventional ionospheric Ohm's law (see Song et al. (2001) and Vasyliūnas and Song (2005) for critical discussions of its derivation and meaning).

The actual approach to steady state is somewhat more complicated than the preceding sketch suggests. In addition to the current in the ionosphere given in Eq. (71), $\delta \boldsymbol{B}$ from deceleration of the plasma also launches a transient Alfvén wave into the magnetosphere, which acts to decelerate the plasma flow in the magnetosphere as well. Only if there are dynamical processes in the magnetosphere that act to maintain the initial flow, launching appropriate Alfvén waves toward the ionosphere in turn, can the velocity difference between the plasma and the neutral atmosphere be sustained in a steady state.

\section{Summary and conclusions}

Although Ampère's law, with the displacement current term neglected, equates $\boldsymbol{J}$ and $(c / 4 \pi) \nabla \times \boldsymbol{B}$, treating both quantities on an equal footing, they can be distinguished, as shown in this paper, by positing an initial state with only one of the two present and then using the basic equations (including the complete Maxwell's equations) to follow the subsequent development of both. By this method, one of the two can be unambiguosly identified as producing the other, with results that depend on the parameter regime. In the ordinary laboratory environment of confined currents surrounded by a vacuum (or the electromagnetic equivalent thereof), $\boldsymbol{J}$ clearly produces $\boldsymbol{B}$. In a plasma that is sufficiently dense so that the electron inertial length $\lambda_{e} \ll \mathcal{L}$ (the length scale of the spatial gradients), on the other hand, $\nabla \times \boldsymbol{B}$ produces $\boldsymbol{J}$, in the precise sense that $\boldsymbol{J}$ changes, if necessary, from its given initial value to become equal to $(c / 4 \pi) \nabla \times \boldsymbol{B}$ (with $\boldsymbol{B}$ not changing significantly from its initial value). The agent of the change in both cases is the electric field of the displacement current term: its effect on $\boldsymbol{B}$ propagates at the speed of light, but in the presence of a free-electron concentration $n_{e}$ it also changes $\boldsymbol{J}$ on the time scale $1 / \omega_{p}$, and the ratio (time to change $\boldsymbol{J}) /($ time to change $\boldsymbol{B})$ is $c / \omega_{p} \mathcal{L} \equiv \lambda_{e} / \mathcal{L}$.

The criterion on spatial scales $\mathcal{L} \gg \lambda_{e}$, together with the criterion on time scales $\tau \gg 1 / \omega_{p}$, has previously (Vasyliūnas, 2005) been shown to define the large-scale plasma regime in which the time evolution of $\boldsymbol{J}$ cannot be calculated directly, but only, through Ampère's law, from the time evolution of $\boldsymbol{B}$, the latter being governed by Faraday's law with the electric field determined (in this largescale plasma regime) by plasma dynamics through the generalized Ohm's law. The present paper extends this conclusion from $\partial \boldsymbol{J} / \partial t$ to $\boldsymbol{J}$ itself: in a fundamental physical sense, $\boldsymbol{J}$ in the large-scale plasma regime is determined by $(c / 4 \pi) \nabla \times \boldsymbol{B}-(1 / 4 \pi)(\partial \boldsymbol{E} / \partial t)$. This has, of course, long been a familiar concept within magnetohydrodynamics (Cowling, 1957; Dungey, 1958), recently emphasized particularly by Parker $(1996,2000)$; what the present paper does is to exhibit in detail how it follows from the basic equations.

The phrase "in a fundamental physical sense" requires some explanation. There are many instances in magnetospheric physics of $\boldsymbol{J}$ being calculated not from $(c / 4 \pi) \nabla \times \boldsymbol{B}$, but in essentially all cases these are currents required in order for $\boldsymbol{J} \times \boldsymbol{B} / \mathrm{c}$ to balance a mechanical stress (e.g. plasma pressure gradient in the magnetosphere, or drag force from the difference between plasma and neutral bulk flows in the ionosphere), under the a priori assumption that stress balance holds. If, to determine how and on what time scale these currents came about, one posits an initial state of the mechanical stress only, without current, and follows the evolution of the system, the result is that at first the stress imbalance produces a change in plasma flow, which deforms the magnetic field, the curl of the deformed field being what finally makes the current; the time scale for the process is typically related to the Alfvén wave travel time.

The physical sequence of what determines what in largescale plasma systems may be summarized as follows:

1. The electric field is determined directly by the generalized Ohm's law (neglecting the $\partial \boldsymbol{J} / \partial t$ term): a combination of flows and kinetic tensors of the various particle species.

2. The time derivative of the magnetic field is determined by the curl of the electric field, hence equivalently by the curl of the generalized Ohm's law, which can be written 
as

$$
\begin{gathered}
\partial \boldsymbol{B} / \partial t=\nabla \times\left\{\boldsymbol{V} \times \boldsymbol{B}-\left(\boldsymbol{J} \times \boldsymbol{B} / n_{e} e\right)-\left(\nabla \cdot \boldsymbol{\kappa}_{e}\right) / n_{e} e\right. \\
\left.+\left[c\left\langle\delta n_{e} \delta \boldsymbol{E}\right\rangle+\left\langle\delta\left(n_{e} \boldsymbol{V}-\boldsymbol{J} / e\right) \times \delta \boldsymbol{B}\right\rangle\right] / n_{e}\right\},
\end{gathered}
$$

a more general approximation than Eq. (60), containing as additional terms (for a plasma containing only electrons and one species of singly charged ions with $m_{i} \gg m_{e}$ ) the electron kinetic tensor and the averaged small-scale fluctuation terms (see Eq. (19) of Vasyliūnas, 2005, and associated discussion).

3. The current density is determined by Ampère's law, Eq. (7) (the displacement current may usually be neglected)

4. In many cases, the dominant term on the right-hand side of Eq. (72) is the $\boldsymbol{V} \times \boldsymbol{B}$ term, the time derivative of which is determined by the plasma momentum equation; the magnetic field can then be visualized as deformed by being bodily carried by the plasma bulk flow (MHD approximation). In the next approximation, the $\boldsymbol{J} \times \boldsymbol{B}$ term is added; the magnetic field is then deformed by the electron bulk flow (Hall MHD approximation). The needed value of $\boldsymbol{J}$ is available from step 3) (and often may be advantageously replaced by $(c / 4 \pi) \nabla \times \boldsymbol{B}$ in the equations). The sequence embodied in steps 1) through 4) remains valid, however, even when additional terms in the generalized Ohm's law, such as electron kinetic tensor or fluctuation correlations, need to be included. Even when the MHD approximation does not apply, any change in $\boldsymbol{V}$ still leads to a change in $\boldsymbol{B}$ - merely one more complicated than a simple deformation by the flow. (That the extra terms in Eq. (72) should change precisely so as to offset the change in $\boldsymbol{V} \times \boldsymbol{B}$ is most improbable!)

The above is the correct description (deduced from analysis of the exact dynamical equations) of the sequence in which the various quantities are physically determined, in the approximation appropriate to large-scale plasmas. The equations of each step also provide mathematical equalities between the quantities. These equalities can, of course, be read in either direction and should not be taken as giving the direction of physical determination, one way or the other. Particularly when dealing with quasi-equilibrium or slowly varying formulations, of which the conventional magnetosphere/ionosphere coupling theory (e.g. Vasyliūnas, 1970, 1972; Wolf, 1983) is a notable example, one must take care not to overlook this distinction between physical determining relation and mere quantitative equivalence.

Acknowledgements. In writing this paper I profited from ideas and suggestions by E. N. Parker concerning a previous publication (Vasyliūnas, 2005). I am grateful to the referees for useful comments that led to clarification of some points.

Topical Editor T. Pulkkinen thanks V. Semenov, N. Tsyganenko and another referee for their help in evaluating this paper.

\section{References}

Cowling, T. G.: Magnetohydrodynamics, Section 1.3, Interscience Publishers, Inc., New York, 1957.

Dungey, J. W.: Cosmic Electrodynamics, Section 1.4, Cambridge University Press, London, 1958.

Heikkila, W. J.: Comment on "The alternative paradigm for magnetospheric physics" by E. N. Parker, J. Geophys. Res., 102, 96519656, 1997.

Lui, A. T. Y.: Electric current approach to magnetospheric physics and the distinction between current disruption and magnetic reconnection, in: Magnetospheric Current Systems, edited by: Ohtani, S.-I., Fujii, R., Hesse, M., and Lysak, R. L., AGU Geophysical Monograph 118, Washington D.C., 31-40, 2000.

Northrop, T. G.: The Adiabatic Motion of Charged Particles, Interscience Publishers, New York, 1963.

Parker, E. N.: Newtonian development of the dynamic properties of ionized gases of low density, Phys. Rev., 107, 924-933, 1957.

Parker, E. N.: The alternative paradigm for magnetospheric physics, J. Geophys. Res., 101, 10587-10625, 1996.

Parker, E. N.: Reply, J. Geophys. Res., 102, 9657-9658, 1997.

Parker, E. N.: Newton, Maxwell, and magnetospheric physics, in: Magnetospheric Current Systems, edited by: Ohtani, S.-I., Fujii, R., Hesse, M., and Lysak, R. L., AGU Geophysical Monograph, 118, Washington D.C., 1-10, 2000.

Song, P., Gombosi, T. I., and Ridley, A. J.: Three-fluid Ohm's law, J. Geophys. Res., 106, 8149-8156, 2001.

Vasyliūnas, V. M.: Mathematical models of magnetospheric convection and its coupling to the ionosphere, in: Particles and Fields in the Magnetosphere, edited by: McCormack, B. M., D. Reidel Publishing Co., Dordrecht-Holland, 60-71, 1970.

Vasyliūnas, V. M.: The interrelationship of magnetospheric processes, in: The Earth's Magnetospheric Processes, edited by: McCormack, B. M., D. Reidel Publishing Co., DordrechtHolland, 29-38, 1972.

Vasyliūnas, V. M.: Time scale for magnetic field changes after substorm onset: constraints from dimensional analysis, in: Physics of Space Plasmas (1995), Number 14, edited by: Chang, T., and Jasperse, J. R., MIT Center for Geo/Cosmo Plasma Physics, Cambridge, Massachusetts, 553-560, 1996.

Vasyliūnas, V. M.: Electric field and plasma flow: What drives what?, Geophys. Res. Lett., 28, 2177-2180, 2001.

Vasyliūnas, V. M.: Time evolution of electric fields and currents and the generalized Ohm's law, Ann. Geophys., 23, 1347-1354, 2005 ,

SRef-ID: 1432-0576/ag/2005-23-1347.

Vasyliūnas, V. M., and Song, P.: Meaning of ionospheric Joule heating, J. Geophys. Res., 110, A02301, doi:10.1029/2004JA010615, 2005.

Wolf, R. A.: The quasi-static (slow-flow) region of the magnetosphere, in: Solar-Terrestrial Physics, edited by: Carovillano, R. L., and Forbes, J. M., D. Reidel Publishing Co., DordrechtHolland, 303-368, 1983. 